\title{
Simulation of Beam Forming Solutions for High Altitude Air Bone Systems
}

\author{
Basuraju S \\ CEC,Benjanapadavu .
}

\author{
Shantharama Rai \\ CEC,Benjanapadavu.
}

\author{
Aaquib Nawaz \\ Ashudeep Infotech,Bengaluru.
}

\begin{abstract}
ABSTRUCT
In order to reduce the traffic problems in mobile communication network, the mobile communication system at base stations should use spatial filtering techniques This can be achieved by using Adaptive antenna system at base station. Adaptive antenna system works on two algorithms DOA and $\mathrm{ABF}$, these algorithms have to be optimized in order to achieve better performance. This paper explains about the Simulation of Beam forming for High Altitude Airborne Systems which involves simulation of ABF, algorithms and comparing their performances.
\end{abstract}

\section{INTRODUCTION}

Beam forming is a signal processing technique used in sensor arrays for directional signal transmission or reception. The spatial selectivity is achieved by using adaptive or fixed receive/transmit beamformer units. Beamforming can be used for both radio or sound waves. It has found numerous applications in Radar, Sonar, Seismology, Wireless Communication, Radio Astronomy, Speech, Acoustics and Biomedicine. Adaptive Beamforming is used to detect and estimate the signal of interest at the output of a sensor array by means of data adaptive spatial filtering and interference rejection.

The main objective of this spatial signal pattern shaping is to simultaneously place a main beam toward the Signal-ofInterest (SOI) and ideally nulls toward directions of interfering signals or Signals Not of Interest (SNOIs).

\section{LITRETURE SURVEY}

The latest advancements in the field of smart antenna Suzanna[1], understanding of Direction of Arrival and Beamforming algorithms are explicitly described in the following papers.George V Tsoulos [2] presents a general overview of smart antenna along with their application and potential benefits for mobile communication systems. It begins with the description of various types of smart antenna Manolakis[3] and then provides information about multiple benefits involved such as increased transmit power and capacity[4]. It reaffirms the fact that exploiting different characteristics of smart antennas can lead to several operational benefits for a communication system.Jack $\mathrm{H}$. Winters [5] in his paper gives an overview about the need for Smart Antennas (SA) in mobile systems. The paper briefs about the various Beamforming and DOA algorithms along with their hardware implementation. Finally the author has concluded the fact that, the implementation of SA has little impact on the physical layer thereby improving the Quality of Service (QoS)[6] and reducing interference at the same time.

\section{ADAPTIVE BEAMFORING:}

Adaptive Beam forming is a technique in which an array of antennas is exploited to achieve maximum reception in a specified direction while rejecting signals of the same/different frequency from other directions. The weights are computed and adaptively updated in real time based on signal samples. The adaptive process permits narrower beams in look direction and reduced output in other directions, which results in significant improvement in Signal to Interference Noise Ratio (SINR).

\subsection{Adaptive Beam forming problem formulation}

Considering a problem of wiener filtering [1], with reference to a non stationary process.

Let $w(n)$ denote unit sample response of the FIR wiener filter that produces minimum mean square estimate of desired signal $s(n)$. The output of the filter is given by

$$
y(n)=\sum_{k=0}^{L} w(k) x(n-k)
$$

Where ' $L$ ' is the order of filter. If $x(n)$ and $s(n)$ are wide sense stationary processes then error signal is given by.

$$
e(n)=s(n)-y(n)
$$

The filter coefficients or beam forming array weights $w(n)$ that minimizes the Mean Square Error $|e(n)|^{2}$ is found by solving the Weiner-Hopf equation given by

$w(n)=R_{x x}^{-1} r_{s x}$

Where, $R_{x x}$ is the autocorrelation of induced signal $x(n)$ and $r_{s x}$ is the cross correlation between reference signal $s(n)$ and induced signal $x(n)$. In many aspects, the design of adaptive Beamformer is much more difficult than design of beam former based on Winters equation[5]. This problem can be simplified by considering the weight update equation to be 
$w(n+1)=w(n)+\Delta w(n)$

Where, $\Delta w(n)$ is the correction applied to calculate new weights. This type of weight updating for $w(n)$ forms the heart of every beamforming algorithm and each of the beamforming algorithms varies in terms of computation of weights.In a practical case, if $s(n)$ is the signal samples corresponding to look direction, $\mathrm{i}(\mathrm{n})$ is interfering signal samples corresponding to jamming directions and $n_{0}(n)$ is noisy signal samples due to receiver components. The induced signal is given by

$$
x(n)=s(n) a\left(\theta_{0}\right)+\sum_{i=1}^{M} i_{i}(n) a\left(\theta_{i}\right)+n_{0}(n)
$$

Where, $\mathrm{M}$ is number of jamming sources, $a\left(\theta_{0}\right)$ is desired steering vector and $a\left(\theta_{i}\right)$ is the steering vector corresponding to $i^{\text {th }}$ interference signal.Since jamming signals (or interfering signals) are of no interest, it is assumed $i(n)_{1}=i(n)_{2} \ldots \ldots=i(n)_{M}=i(n) \quad$ with this modification equation (3.5) can be written as

$x(n)=a\left(\theta_{0}\right) s(n)+i(n) \sum_{i=1}^{M} a\left(\theta_{i}\right)+n_{0}(n)$

In matrix notation, induced signal can be written as

$$
X=A_{\theta_{0}} S+A_{i n} I_{i}+N
$$

Where,$X$ represents $\mathrm{L} \times \mathrm{N}_{\mathrm{s}}$ induced signal matrix, $N_{s}$ is total number of samples, ' $L$ ' represents number of array elements, ' $S$ ' represents reference signal samples. $A_{\theta_{0}}$ is desired steering vector of order Lx1, $\boldsymbol{I}_{\boldsymbol{i}}$ represents interference signal samples matrix of order $1 \mathrm{xN}_{\mathrm{s}}$, $N$ represents Gaussian noise matrix of order $\mathrm{LxN}_{\mathrm{s}}$ and $\boldsymbol{A}_{\text {in }}$ is Lx1 column vector that is obtained by adding all columns of array manifold vector as shown (3.8)

$A_{i n}=\left[\begin{array}{c}1 \\ e^{i x_{1}} \\ \vdots \\ e^{i y_{1}}\end{array}\right]+\left[\begin{array}{c}1 \\ e^{i x_{2}} \\ \vdots \\ e^{i y_{2}}\end{array}\right]+\ldots \ldots \ldots \ldots \ldots . .\left[\begin{array}{c}1 \\ e^{i x_{m}} \\ \vdots \\ e^{i y_{m}}\end{array}\right]_{(3.8)}$

$\mathrm{X} 1=2 \pi \mathrm{d} \sin \theta_{1}, \mathrm{X} 2=2 \pi \mathrm{d} \sin \theta_{2}, \mathrm{Xm}=2 \pi \mathrm{nd} \sin \theta_{m}$

$\mathrm{Y} 1=2 \pi \mathrm{d}(\mathrm{L}-1) \sin \theta_{1}, \mathrm{Y} 2=2 \pi \mathrm{d}(\mathrm{L}-1) \sin \theta_{2}$

$\mathrm{Ym}=\mathrm{Ym}=2 \pi \mathrm{d} \sin \theta_{m}$
Where, ' $\mathrm{d}$ ' is the distance between antenna elements, $\theta_{1}, \theta_{2}, \ldots \ldots ., \theta m$ are directions of jamming signals and $\mathrm{M}$ is number of jamming signals.

\section{LEAST MEAN SQUARE (LMS)}

The LMS algorithm [1] is the most widely used adaptive beamforming algorithm, being employed in several communication applications. It has gained popularity due to its low computational complexity and proven robustness. The LMS algorithm changes the weight vector $w(n)$ along the direction of the estimated gradient based on the steepest descent method[2]. In employing the LMS algorithm, it is assumed that sufficient knowledge of the reference signal is present.

\subsection{Derivation of LMS weight vector}

The weight update equation of Steepest decent method [2] is given by

$$
\begin{aligned}
& \mathrm{W}(\mathrm{n}+1)=\mathrm{w}(\mathrm{n})+\frac{\mu l}{\|x\|} \\
& w(n+1)=w(n)+\mu E\left\{e(n) x^{*}(n)\right\}
\end{aligned}
$$

Where, $E$ is the expectation operator, $\mu$ is the step size used for convergence of beam forming algorithm, $e(n)$ is the error signal and $x^{*}(n)$ is the conjugate of induced signal.A practical limitation with steepest decent method is that $E\left\{e(n) x^{*}(n)\right\}$ is generally unknown. Therefore, it must be replaced with an estimate such as sample mean given by

$$
E\left\{e(n) x^{*}(n)\right\}=\frac{1}{L} \sum_{l=0}^{L-1} e(n-l) x^{*}(n-l)
$$

Incorporating this estimate as in equation (3.11) into steepest decent algorithm, the update for $w(n)$ is given by

$$
\begin{aligned}
& w(n+1)=w(n)+\mu E\left\{e(n) x^{*}(n)\right\} \\
& =w(n)+\mu \frac{1}{L} \sum_{l=0}^{L-1} e(n-l) x^{*}(n-l)
\end{aligned}
$$

A special case of equation (3.12) occurs if we use a one point sample mean, i.e $(l=1)$ as given by

$$
E\left\{e(n) x^{*}(n)\right\}=e(n) x^{*}(n)
$$

Using the condition of equation (3.13), the weight vector update equation assumes a particular simple form as given by

$$
w(n+1)=w(n)+\mu e(n) x^{*}(n)
$$

Where, $\mu$ is the step size which can be in the range given by

$$
\mathrm{O} \leq \mu \leq \frac{2}{3 \operatorname{tr}\left(R_{x x}\right)}
$$


Where, $\operatorname{tr}\left(R_{x x}\right)$ is the trace of auto correlation matrix.For simulation, step size is given by

$$
\mu=\frac{2}{3 \operatorname{tr}\left(R_{x x}\right)}
$$

The advantage of using LMS algorithm over steepest decent method is that the expectation operator in steepest decent method is removed from weight vector by considering a single sample.

\section{VARIABLE STEP SIZE LMS (VSS- LMS)}

The Least Mean Square (LMS) algorithm[1] is one of the most popular algorithms in adaptive signal processing, due to its simplicity and robustness. Many different modifications were proposed to improve performance of the LMS and a large number of results on its steady state misadjustment and its tracking ability has been obtained. Unfortunately[2], its convergence rate is highly dependent on the conditioning of autocorrelation matrix. When inputs are highly correlated, convergence rate degrades radically. Variable Step-Size LMS (VSS LMS) algorithms are used[3], with the intention of decreasing misadjustment and to maximize convergence rate. Step size is larger when the estimate is far from the optimum value and a smaller step-size as it approaches the optimum value. The performance of this method is promising especially in non stationary environment[3].

The autocorrelation matrix is given by

$$
R=E\left[x(n) x(n)^{H}\right]
$$

$$
\text { Where, } x(n) \text { is the induced signal and } x(n)^{H} \text { is }
$$

the hermitian transpose of $x(n)$

The step size is calculated, during each iteration by using equation

$$
\mu(n+1)=\alpha \mu(n)+\gamma|e(n)|^{2}
$$

Where, ' $\alpha$ ' indicates the correlation of the present step size to the previous step size, $\alpha$ is in the range $0<\alpha<1$ and $\gamma$ is used to control convergence characteristics of VSS-LMS algorithm, $\gamma=0.5$ and $\mathrm{e}(\mathrm{n})$ is given by

$$
e(n)=d(n)-y(n)
$$

Where, $d(n)$ is the reference signal and $y(n)$ is the array output. The upper bound on the step size is given by [7]

$$
\mu_{\text {upper }}=\frac{2}{3 \operatorname{tr}(\operatorname{cov}(x))}
$$

Where, $\operatorname{tr}(\operatorname{cov}(x))$ is the trace of covariance matrix.

The weight update equation to put main beam in the desired direction and nulls in the jammer directions using VSS-LMS is given by

$$
w(n+1)=w(n)+\mu(n+1) e(n) x(n)
$$

The algorithm is bounded in step size with upper bound as defined in equation (4.4). During each iteration the step size is changed as

$$
\begin{aligned}
\mu(n+1) & =\mu_{\text {upper }} \quad \text { if } \quad \mu(n+1)>\mu_{\text {upper }} \\
& =0 \quad \text { if } \quad \mu(n+1)<0 \\
& =\mu(n+1) \quad \text { otherwise }
\end{aligned}
$$

\section{GRIFFITHS' ALGORITHM.}

Griffiths' algorithm[6] utilizes certain a priori knowledge (when available) to create an effective real time adaptation process. The algorithm can be used for a number of applications including Noise Control, Adaptive Beamforming and Acoustic Signal Processing. The algorithm is ideal when the cross-correlation between the desired response and the input signal vector (i.e. the inputs to the weights) are known a priori. In this case, the algorithm can be executed without the need for a real time response input $s(n)$ [5].

The cross correlation $r_{s x}$ between the desired signal $\mathrm{d}(\mathrm{n})$ and induced signal $\mathrm{x}(\mathrm{n})$ is given by

$$
r_{s x}=\sum_{n=1}^{S_{S}} d(n) x(n)
$$

Where, ' $d(n)$ ' is desired signal samples, $S_{\mathrm{s}}$ is number of samples and $\mathrm{x}(\mathrm{n})$ is induced signal samples.

The weight update equation for Griffith's algorithm can be derived by using LMS algorithm, which is given by

$$
\begin{aligned}
w(n+1)= & w(n)+2 \mu e(n) x(n) \\
& =w(n)+2 \mu(d(n)-y(n)) x(n) \\
& =w(n)+2 \mu r_{s x}-2 \mu y(n) x(n)
\end{aligned}
$$

Where, $\mu$ is the step size, $r_{s x}$ is the cross- correlation and $y(n)$ is the array output.

\section{VARIABLE STEP SIZE GRIFFTHS' ALGORITHM (VSSG)}

The Variable Step Size Griffiths' algorithm [4] is a combination of the Variable Step Size LMS algorithm and the Griffiths' algorithm. The algorithm is expected to combine the merits of the Variable Step Size LMS algorithm and Griffiths' algorithm. The motivation behind this algorithm is, the use of an algorithm which would achieve faster convergence through the use of a variable step size LMS algorithm and a smoother gradient through the use of the Griffiths' algorithm[5]. The use of a common gradient would decrease the computational complexity, without compromising the efficiency of the resulting algorithm. However, implementation indicates that the algorithm's performance degraded with the dependence of the step size on the same gradient as used for the weight updating[4].

The weight update equation in case of VSSG algorithm is given by 
$\mathrm{W}(\mathrm{n}+1)=\mathrm{w}(\mathrm{n})+\frac{\mu l}{\|x\|}$

\section{RESULTS}

Table 1: Less Antenna Elements and Single Jammer

\begin{tabular}{|l|l|l|l|}
\hline Algorithm & $\begin{array}{l}\text { Desired } \\
\text { Angle }\end{array}$ & $\begin{array}{l}\text { Jammer } \\
\text { Angle }\end{array}$ & $\begin{array}{l}\text { No Of } \\
\text { Antenna } \\
\text { Elements }\end{array}$ \\
\hline LMS & 45 & 5 & 8 \\
\hline VSS-LMS & 45 & 10 & 8 \\
\hline Griffiths & 65 & 6 & 8 \\
\hline VSSG & 70 & 5 & 8 \\
\hline
\end{tabular}

\section{LMS:}

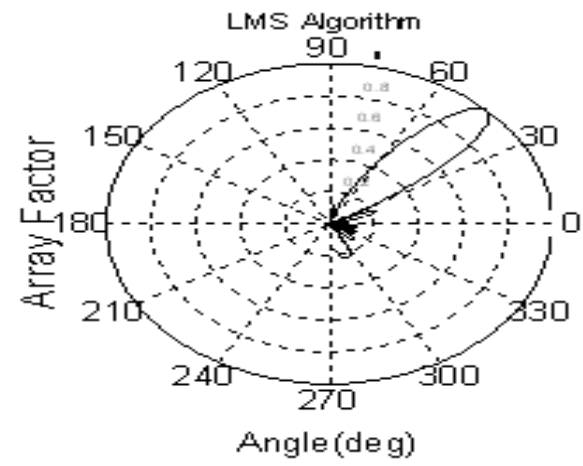

Fig1: Polar Plot for LMS Algorithm.

Fig1 shows that the LMS algorithm is capable of forming main beam at desired direction of 45 degree with less antenna elements

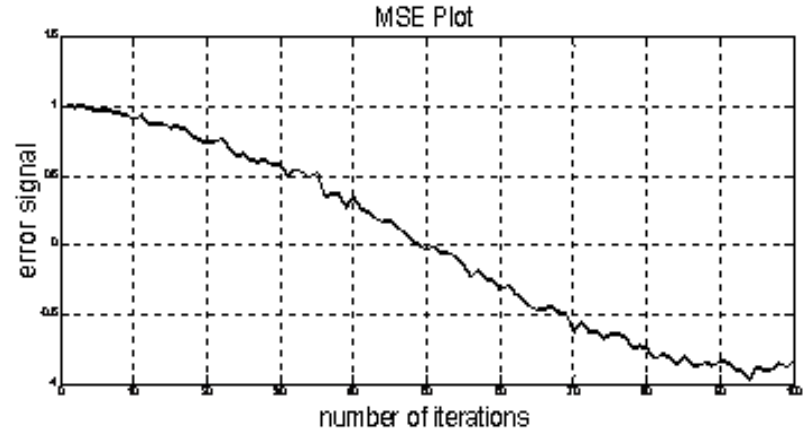

Fig3:MSE Plot for LMS Algorithm.

Fig3 shows the mean square error variation for LMS algorithm for 100 iterations

\section{Variable Step Size LMS:}

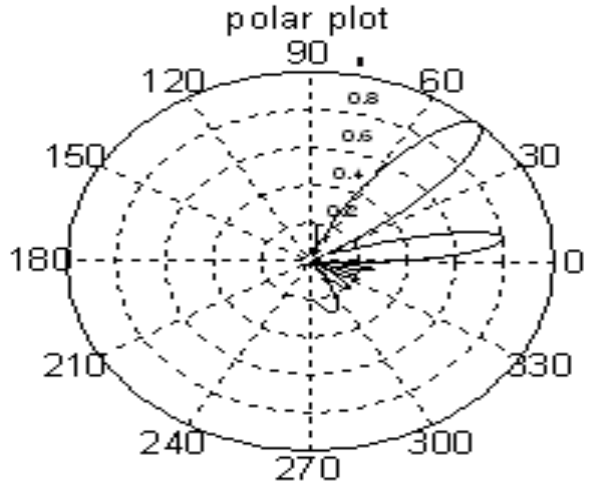

Fig3: Polar Plot for VSSLMS Algorithm.

Fig3 shows that the VSSLMS algorithm is capable of forming main beam at desired direction of 45 degree with less antenna elements



Fig4:MSE Plot for VSS-LMS Algorithm.

Fig4 shows the mean square error variation forVSS- LMS algorithm for 100 iterations

\section{Griffiths:}

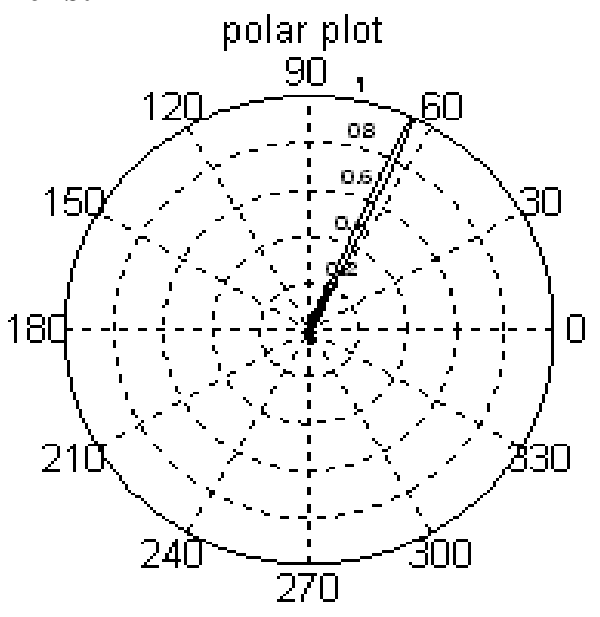

Fig5: Polar Plot for Griffiths Algorithm

Fig5 shows that the Griffiths algorithm is capable of forming main beam at desired direction of 65 degree with less antenna elements. 


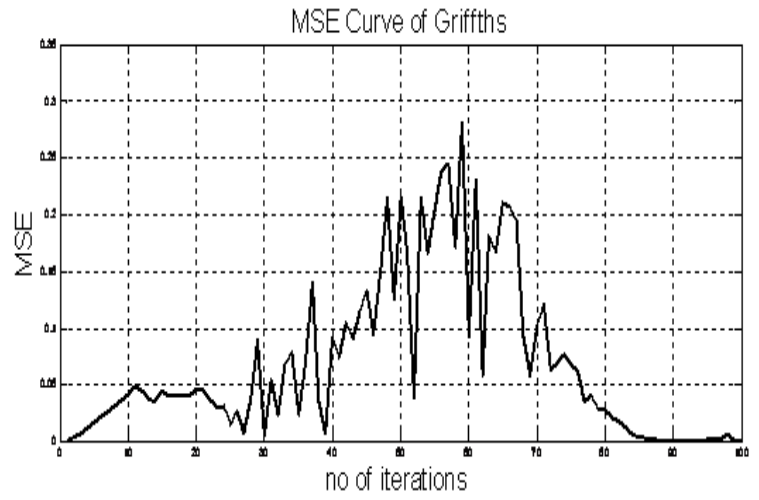

Fig6:MSE Plot for Griffiths Algorithm.

Fig6 shows the mean square error variation for Griffiths algorithm for 100 iterations.

\section{VSSG:}

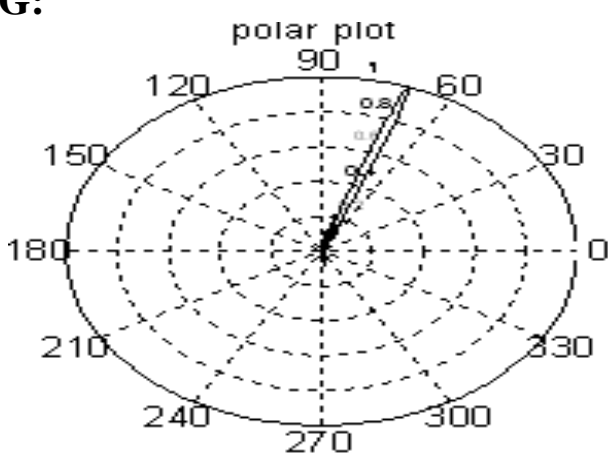

Fig7: Polar Plot for VSSG Algorithm

Fig7 shows that the VSSG algorithm is capable of forming main beam at desired direction of 70 degree with less antenna elements



Fig8:MSE Plot for VSSG Algorithm.

Fig6 shows the mean square error variation for VSSG algorithm for 100 iterations.

\section{CONCLUTIONS}

In LMS Algorithm problem is Low Convergence, To overcome we go for VSSLMS. Here we achieve high Convergence. In order to get high Convergence in VSS-LMS, The error rate will increase. so in order decrease error or noise we will go for Griffths Algorithm. Variable Step Size Griffiths' (VSSG)This is combination of VSS-LMS and Griffths here High convergence and Low error or noise,VSSG is best because here we get High convergence and Low error or noise.

\section{FUTURE SCOPE}

Beam forming is a technology that provides wireless networks with less latency and high data rates. Some of the disadvantages of this project can be overcome by extension of this project work which are listed as

1. Square Array can be utilized to steer the beam over a wider range of angles for both azimuth and elevation.

2. Direction of Arrival algorithms like Root MUSIC (MUltiple Signal Classification) and ESPRIT (Estimation of Signal Parameters via Rotational Invariance Techniques) algorithmscan be implemented to obtain high resolution.

3. The RF cost of beam forming can be reduced by utilizing Dielectric Lens in front of antenna array which increases directivity. The Directivity obtained by using dielectric lens can be achieved by using large amount of antenna elements in the array structure but large array increasing the complexity and cost.

4. Most of the noise considered for practical purposes is white noise. However, in nature we observe that additive white Gaussian noise is pre-dominant. Thus, measures have to be taken to take this constraint into account.

5. Other techniques like Neural Networks can be employed to reduce the computational burden on the existing Adaptive Beam forming techniques.

6. To increase the capacity of the links (channels), new technologies like Multiple Input Multiple Output (MIMO) can be used, along with the smart antennas, to provide higher channel capacity

\section{REFERENCES}

[1]. Suzanna LaMar1,2, Hugh Nguyen1, and Paul Zavidniak1,Beamforming Solutions for Interference Reduction for High Altitude Airborne CDMA Systems .IEEE-2011.

[2]. George V Tsoulos , "Smart antennas for mobile communication systems: Benefits and Challenges", IEEE proceedings of international conference on information technology, Vol-11, Issue-2 ,pp 84-94.

[3]. D. G. Manolakis, Vinay K Ingle and Stephen M Kogon, "Statistical and Adaptive Signal processing spectral estimation, signal modeling, Adaptive filtering and array processing," Mc Graw Hill 2000.

[4]. Seungwon Choi, Hong-Min Son, and Tapan K sarkar, "Implementation of a Smart Antenna System on a General-Purpose Digital Signal Processor Utilizing a Linearized CGM", Digital Signal Processing Journal, July-1997, Vol-7, pp 105-119.

[5]. Jack H. Winters , "Smart Antennas for Wireless Systems", proceedings of IEEE international conference on signal processing, June 2005, pp 107-109

[6]. Sungsoo, Seungwon and Tapan K sarkar, "An Adaptive Beamforming Algorithm with a Linear Complexity for a Multipath Fading CDMA Channel", IEICE Transaction. 